\title{
ANALYTICAL SOLVING A NONLINEAR MODEL OF THE GLOW DISCHARGE
}

\author{
M. ARAM
}

\begin{abstract}
Tehran, Iran, Postal Code: 13938-84368*
\end{abstract}
mjaram200003@gmail.com

\begin{abstract}
A nonlinear model has been introduced for the positive column of DC glow discharge in a pure sealed, or low flow, gas media by including the diffusion, recombination, attachment, detachment, process and having the two-step ionization process of the metastable excited states, too. By the combination of the system of the nonlinear continuity equations of the system, using some physical estimations, and degrading the resulted nonlinear PDE in polar and rectangular systems of coordinate the steady-state nonlinear ODE have been derived. Using a series-based solution, an innovative nonlinear recursion relation has been proposed for calculating the sentence of series. Using the state of elimination of free charge on the outer boundary of the discharge vessel, the universal equation of the characteristic energy of the electrons versus the similarity variable, using the maximum degree of ionization as the parameter, has been derived.
\end{abstract}

Keywords: CW glow discharge, nonlinear differential equation, pure gas environment..

\section{Introduction}

Electric discharge in gases [1-3] has played a significant role in the emergence and development of modern physics, and in addition to its many applications in science and technology [4-7], it remains a field for experimental and theoretical research [8-10]. The glow discharge is very important in a wide range from glow discharge lamps [11] to energy injection in continuous and pulsating gas lasers such as carbon dioxide [12] and excimer [13] laser. Since the optimization of small signal gain and overall efficiency of these lasers is strongly dependent on the discharge conditions, so it requires further theoretical understanding in this area. It has been already discovered, in the positive column (PC) of the glow discharge (GD), there is an equation between electron characteristic energy, $k T_{e}$, and the similarity variable, $n R$ or $p R$ where $n$ is overall particle density of media, $p$ is overall pressure of the media and $\mathrm{R}$ is the internal radius of the discharge vessel, no matter it is DC or AC discharge [14-17]. A basic analytical formula for this equation is introduced by most of the references which have been derived. But the proposed physical model has been based on the diffusion dominated PC of the GD, and the second order ionization process, i.e., collisional and photo-ionization of the metastable states, are ignored.

In this paper, results of an investigation on the generalized equation of the characteristic electron energy $k T_{e}$ on the similarity variable of the glow discharge $N R$, where $N$ is the total particle density of the media and $\mathrm{R}$ is the internal radius of the discharge vessel, have been reported. The process of the dif-

* Retired of the Photonics and quantum technologies research school, N.S.T.R.I, Tehran, IRAN. fusion, recombination, and ionization of the ground and metastable excited states, by the electrons and photons, has been included in the model because the intention was for the model to be, a complete picture of the all glow discharges as more as possible, including sub-normal to abnormal. Obviously the model has to have nonlinearity [18]. The physical model has been based on the time-independent system of the continuity equations of the electron and ions (positive and negative, assuming negative ion density is negligible), in the glow discharge of pure gases, or metal vapor. The terms of particle drift and diffusion are included in the left hand side. The ionization (collisional $1^{\text {st }}$ and $2^{\text {nd }}$ order) and electron-ion recombination have been included in the right hand side as is described in the next section.

It has to be noted here that no other theoretical results have been found for comparison. Only there is an experimental work which has been done on the media of the glow discharge of the $\mathrm{CO}_{2}$ laser [19] and shows the dependence of the $k T_{e}$ to the similarity variable using the electron density as the parameter and could be imagined as an strong motivation for doing this research.

\section{Physico - Mathematical considerations and analytic formulas}

Starting with the introduction system of the timeindependent continuity equations for electrons and ions (positive \& negative) in a continuous glow discharge in pure gas and/or metal vapor environment. Although, the effect of the gas flow on the current density is ignored here by restricting the applied condition to sealed off or low gas flow medias, the result isn't affected principally by such a simplification. By 
taking into account a first-order (linear) and secondorder (non-linear) terms, the system of the equations is as follows:

$$
\begin{aligned}
\nabla \cdot \mathbf{J}_{\mathrm{e}} & =\left(n R_{\mathrm{ion}}-n R_{\mathrm{att}}+n_{-} R_{\mathrm{det}}\right) n_{\mathrm{e}} \\
& +n_{\mathrm{m}}\left(R_{\mathrm{ion}}^{\mathrm{m}} n_{\mathrm{e}}+R_{\mathrm{phion}}^{\mathrm{m}} N_{\mathrm{ph}}\right)-n_{+} \gamma_{\mathrm{rec}}^{\mathrm{e}} n_{\mathrm{e}} \\
\nabla \cdot \mathbf{J}_{+} & =n R_{\text {ion }} n_{\mathrm{e}}+n_{\mathrm{m}}\left(R_{\mathrm{ion}}^{\mathrm{m}} n_{\mathrm{e}}+R_{\mathrm{phion}}^{\mathrm{m}} N_{\mathrm{ph}}\right) \\
& -n_{+}\left(\gamma_{\mathrm{rec}}^{\mathrm{e}}\right) n_{\mathrm{e}}-n_{+}\left(\gamma_{\mathrm{rec}}^{ \pm}\right) n_{-} \\
\nabla \cdot \mathbf{J}_{-} & =\left(n R_{\mathrm{att}}+n R_{\mathrm{det}}\right) n_{\mathrm{e}}-n_{+}\left(\gamma_{\mathrm{rec}}^{ \pm}\right) n_{-} \\
\frac{\mathrm{d} n_{\mathrm{m}}}{\mathrm{d} t} & =n R_{\mathrm{exc}}^{\mathrm{m}} n_{\mathrm{e}}+n_{\mathrm{m}}\left(R_{\mathrm{ion}}^{\mathrm{m}} n_{\mathrm{e}}+R_{\mathrm{phion}}^{\mathrm{m}} N_{\mathrm{ph}}\right) \\
& -n_{\mathrm{m}} R_{\mathrm{S} . e l a s}^{\mathrm{m}} n_{\mathrm{e}} .
\end{aligned}
$$

The other decay process of the metastable states in equation (4), esp. the non-radiative decay, are ignored as an estimation, unless the model gets very complicated and nonlinearity gets degree of 3 .

$$
\begin{aligned}
& \frac{\mathrm{d} N_{\mathrm{ph}}}{\mathrm{d} t}=\frac{n_{\mathrm{exc}}}{\tau}-n_{\mathrm{m}} R_{\mathrm{phion}}^{\mathrm{m}} N_{\mathrm{ph}}, \\
& \frac{\mathrm{d} n_{\mathrm{exc}}}{\mathrm{d} t}=n R_{\mathrm{exc}}^{\mathrm{m}} n_{\mathrm{e}}-\frac{n_{\mathrm{exc}}}{\tau} .
\end{aligned}
$$

It should be noted that in relations (4) to (6), the phrase related to radial diffusion is ignored and the time dependence is almost zero, which will be emphasized later. Here $n$, is overall particle density. $n_{\mathrm{e}}, n_{+}$ and $n_{-}$are the density of the electron, positive and negative ions, respectively. $N_{\mathrm{ph}}$ is the overall photon density in the media. $\mathbf{J}_{\mathrm{e}}=-\mu_{\mathrm{e}} \mathbf{E} n_{\mathrm{e}}-D_{\mathrm{e}} \nabla n_{\mathrm{e}}, \mathbf{J}_{+}=$ $n_{+} \mathbf{E} \mu_{+}-D_{+} \nabla n_{+}$, and $\mathbf{J}_{-}=-n_{-} \mathbf{E} \mu_{-}-D_{-} \nabla n_{-}$, are the current densities of the three major species. $E$, $\mu_{\mathrm{e}}, \mu_{ \pm}, D_{\mathrm{e}}$ and $D_{ \pm}$are electric field intensity, mobility of the electron and ions(positive \& negative), and diffusivity of the electron and ions (positive \& negative), respectively. After that, $n_{\mathrm{m}}, n_{\mathrm{exc}}, \tau$ and $N_{\mathrm{ph}}$ are the overall density of the particles in metastable excited states, density and radiation life time of excited states and overall photon density, respectively. On the other hand, $R_{\text {ion }}, R_{\text {att }}, R_{\text {det }}, R_{\text {ion }}^{\mathrm{m}}, R_{\text {phion }}^{\mathrm{m}}$, $R_{\mathrm{s}-\mathrm{elas}}^{\mathrm{m}}, \gamma_{\text {rec }}^{\mathrm{e}}$ and $\gamma_{\text {rec }}^{ \pm}$are the collisional ionization rate, electron attachment rate, electron detachment rate, collisional ionization rate of the metastable excited states, photoionization rate of the metastable excited states, deactivation rate of the metastable states with super-elastic collision by electron, electron-ion (positive) recombination rate and recom bination rate of the ions (positive \& negative), respectively. At this step, it has to be implemented that there is dependence to the reduced electric field $(E / N)$ for all of the plasma media kinetics parameters, like mobility and diffusion. But the interesting point is that $T_{\mathrm{e}}$ and $E / N$ are related [2] too, so we can use the electron temperature as the basic parameter and left a $E / N$ dependent model to the future. The main benefits of such approach is that the basic purpose of the research, where is investigation on functionality of the electron temperature of the similarity variable (in a generalized nonlinear model of glow discharge), is satisfied without being captured by the calculation of the collision integrals, where are required for having relation to the $E / N$.

Now, the equations (1) to (3) have to be combined using the following estimations (notice: the radial diffusion of the negative ions is ignored):

$$
\text { a) } n_{-}<n_{\mathrm{e}} \& \mu_{-} \ll \mu_{\mathrm{e}} \Rightarrow \mathbf{J}_{-} \ll \mathbf{J}_{\mathrm{e}}
$$$$
\Rightarrow \mathbf{J}_{\mathbf{e}}+\mathbf{J}_{-} \approx \mathbf{J}_{\mathrm{e}}
$$

b) $\nabla \mathbf{J}_{-}=0$

$$
\begin{aligned}
& \Rightarrow\left(n R_{\mathrm{att}}-n_{-} R_{\mathrm{det}}\right) n_{\mathrm{e}}=n_{+}\left(\gamma_{\mathrm{rec}}^{ \pm}\right) n_{-} \\
& \approx n_{\mathrm{e}}\left(\gamma_{\mathrm{rec}}^{ \pm}\right) n_{-} \\
& \Rightarrow n_{-} \approx n\left(\frac{R_{\mathrm{att}}}{R_{\mathrm{det}}+\gamma_{\mathrm{rec}}^{ \pm}}\right)=n \varepsilon \\
& \& n_{+}=n_{\mathrm{e}}+n_{-}=n_{\mathrm{e}}+n \varepsilon,
\end{aligned}
$$

where $\varepsilon=\frac{R_{\text {att }}}{R_{\text {det }}}+\gamma_{\text {rec }}^{ \pm}$.

By combining (4) to (6) and using the steady-state condition the following relation for the estimation for the number density of particles in the metastable states, has been derived:

$$
\begin{aligned}
\frac{\mathrm{d} n_{\mathrm{m}}}{\mathrm{d} t} & =0 \\
\Rightarrow n_{\mathrm{m}} & =n \frac{R_{\text {exc }}^{\mathrm{m}}}{\left(R_{\mathrm{ion}}^{\mathrm{m}}+R_{\mathrm{S} . \text { elas }}^{\mathrm{m}}+R_{\text {exc }}\right)}=n \varepsilon_{\mathrm{m}},
\end{aligned}
$$

where $\varepsilon_{\mathrm{m}}=\frac{R_{\mathrm{exc}}^{\mathrm{m}}}{\left(R_{\mathrm{ion}}^{\mathrm{m}}+R_{\mathrm{S} \text { elas }}^{\mathrm{m}}+R_{\mathrm{exc}}\right)}$. By inserting the relations (7a), (7b) and (8) in the Equations (1) and (2), the following relation has been derived:

$$
\text { a) } \begin{aligned}
\nabla \cdot \mathbf{J}_{\mathrm{e}} & =-\left(n_{\mathrm{e}} \mu_{\mathrm{e}}+n \varepsilon \mu_{-}\right) \nabla \cdot \mathbf{E}-D_{\mathrm{e}} \nabla^{2} n_{\mathrm{e}} \\
& =\left[n\left(R_{\text {ionizexc }}-\gamma_{\mathrm{rec}}^{\mathrm{e}, \pm}\right)\right] n_{\mathrm{e}}-\gamma_{\mathrm{rec}}^{\mathrm{e}} n_{\mathrm{e}}^{2}
\end{aligned}
$$

b) $\nabla \cdot \mathbf{J}_{+}=\left(n_{\mathrm{e}}+n \varepsilon\right) \mu_{+} \nabla \cdot \mathbf{E}-D_{+} \nabla^{2} n_{+}$

$$
=\left[n\left(R_{\text {ionizexc }}-\gamma_{\text {rec }}^{\mathrm{e}, \pm}\right)\right] n_{\mathrm{e}}-\left(\gamma_{\mathrm{rec}}^{\mathrm{e}}\right) n_{\mathrm{e}}^{2}
$$

where $R_{\text {ionizexc }}=\left(R_{\mathrm{ion}}^{\mathrm{m}}+R_{\mathrm{exc}}^{\mathrm{m}}+\varepsilon_{\mathrm{m}} R_{\mathrm{S} \text {.elas }}^{\mathrm{m}}\right)$ is defined as the overall ionization and excitation rates to metastable states and $\gamma_{\text {rec }}^{\mathrm{e}, \pm}=\gamma_{\text {rec }}^{\mathrm{e}}+\varepsilon \gamma_{\text {rec }}^{ \pm}$is the overall recombination rate.

Next, using the formula (7b) and replacing $R_{\text {ionizexc }}-\gamma_{\text {rec }}^{\text {e, } \pm}$ by $R_{\text {eff }}$, (be notified that $\nabla^{2} n_{+}=$ $\left.\nabla^{2}\left(n_{\mathrm{e}}+n_{-}\right) \approx \nabla^{2} n_{\mathrm{e}}\right)$, equations (9a) and (9b) has been mixed and partial nonlinear equation of the space-dependent charge density in the glow discharge has been derived as follows:

$$
D_{\mathrm{a}}^{\mathrm{e}, \pm} \nabla^{2} n_{\mathrm{e}}+\left(\gamma_{\mathrm{rec}}^{\mathrm{e}}\right) n_{\mathrm{e}}^{2}=0
$$


where, at the right hand has been included in first and second order terms, in aspect of dependence to the electron density. Also, $D_{a}^{e^{ \pm}}$is the unified ambipolar diffusion coefficient that has been defined as follows:

$$
D_{\mathrm{a}}^{\mathrm{e}, \pm}=\frac{D_{+} \mu_{\mathrm{e}}^{\mathrm{eff}}+\mu_{+}^{\mathrm{eff}} D_{\mathrm{e}}}{\mu_{\mathrm{e}}^{\mathrm{eff}}+\mu_{+}^{\mathrm{eff}}} .
$$

Here $\mu_{\mathrm{e}}^{\mathrm{eff}}=\mu_{\mathrm{e}}\left(1+\frac{\varepsilon}{\overline{D \cdot I}} \frac{\mu_{-}}{\mu_{\mathrm{e}}}\right)$ and $\mu_{+}^{\mathrm{eff}}=$ $\mu_{+}\left(1+\frac{\varepsilon}{\overline{D \cdot I}}\right)$ are proposed as effective mobility of the electron, and effective mobility of the positive ions, respectively. Also, $\overline{D \cdot I}=\frac{\bar{n}_{\mathrm{e}}}{n}$ is proposed as the radial average degree of ionization. Simplicity has been tried in definition of the $D_{\mathrm{a}}^{\mathrm{e}, \pm}$, although there is a more complicated formulas for ambipolar diffusion coefficient in mixed gaseous media [20].

It is important to notifie the criteria for having a selfsustained glow discharge i.e. $R_{\text {eff }}=R_{\text {ionizexc }}-\gamma_{\text {rec }}^{\text {e, } \pm}>0$ which obviously put a lower limit on the electron temperature. From empirical evidence, it is reasonable to assume $\beta>\eta^{2} n_{\mathrm{e}}$, which means that the discharge is self sustained. For simplicity, the equation (10) has been written as follows:

$$
\nabla^{2} n_{\mathrm{e}}+\beta n_{\mathrm{e}}-\eta^{2} n_{\mathrm{e}}^{2}=0 .
$$

In the above equation $\beta=\frac{\gamma_{\mathrm{rec}}^{\mathrm{e}}}{D_{\mathrm{a}}^{\mathrm{e}, \pm}}$ and $\eta^{2}=\frac{n R_{\mathrm{eff}}}{D_{\mathrm{a}}^{\mathrm{e}, \pm}}$ are defined as new parameters. The important point is that the physical dimensions of $\beta$ and $\eta^{2}$ are $L^{-2}$ and $\mathrm{L}$ respectively ( $\mathrm{L}$ represents the dimension of length in the physical dimension apparatus).

\section{Solving the nonlinear equation and extracting results}

In this section using the two dimensional Cylindrical and Cartesian system of coordinates, equation (12) has been decomposed into non-linear ordinary differential equation and the general functions of spatial variations of electron density have been derived.

\subsection{Deriving the differential equation in the Cylindrical and Cartesian system of coordinates}

\subsubsection{Cylindrical coordinate}

Assuming $z$-direction as the direction of the electric field intensity and the axis of symmetry of the discharge. So, electron density would be assumed independent of the $\mathrm{z}$, and $\phi$, with a decrease in radial dependence. Using simple mathematical operations, the equation (12) above changes to a nonlinear ordinary second-order differential equation:

$$
\frac{\mathrm{d}^{2} n_{\mathrm{e}}}{\mathrm{d} r^{2}}+\frac{1}{r} \frac{\mathrm{d} n_{\mathrm{e}}}{\mathrm{d} r}+\beta n_{\mathrm{e}}-\eta^{2} n_{\mathrm{e}}^{2}=0,
$$

where $r$ is the cylindrical radial coordinate. After mathematical operations, the equation (14) will result as follows:

$$
\frac{\mathrm{d}^{2} f(r)}{\mathrm{d} r^{2}}+\frac{1}{r} \frac{\mathrm{d} f(r)}{\mathrm{d} r}-\frac{\beta}{2}\left(f^{2}(r)-1\right)=0
$$

In the equation (13), by attention to the dimension of $\beta$ and $\eta^{2}$, it is clear that $f(r)=\left(\frac{2 \eta^{2} n_{\mathrm{e}}(r)}{\beta}-1\right)$ is a dimensionless function of $r$. From the mathematical point of view, this is a nonlinear ordinary differential equation which is very similar to the Bessel ordinary differential equation. There are classic [21] and new [22-28] references about the nonlinear differential equations (partial and ordinary) in physics and mathematics. It is still a fascinating area for research on finding analytical solution, where be simple and general straightforward. At this point, using a generalization of the Frobenius [14] method, a series based formula has been proposed as the analytical solution of the equation (14) as follows:

$$
f(r)=\sum_{n=0}^{\infty} a_{n} r^{n}
$$

By inserting the (15) in the equation (14) and some mathematical operations an innovative, nonlinear recursion formula has been found which, accompanied by the (physical) initial condition, act as the nonlinear generator of the series sentences as follows:

$$
\begin{aligned}
& \text { a) } a_{0}=\frac{2 \eta^{2}\left(n_{\mathrm{e}}\right)_{\max }}{\beta}-1=\frac{4+\sqrt{16+\beta^{2}}}{\beta}, a_{1}=0 \\
& \text { b) } a_{n+2}=\frac{\beta / 2}{(n+2)^{2}} b_{n}, \quad b_{n}=\sum_{j=0}^{n} a_{n-j}^{*} a_{j}
\end{aligned}
$$

where $n=1,2,3, \ldots$

Using the relations (14) to (16), it has been found that the following mathematical replacement for the $f(r)$ is more useful. Science shows the dimensionless property of the series very clearly:

$$
\begin{aligned}
f(r) & =F\left(\beta r^{2}\right)=a_{0}+\sum_{k=1}^{\infty} \frac{b_{2 k-2}}{4(k+1)^{2}}\left(\frac{\beta r^{2}}{2}\right)^{k}, \\
a_{1} & =a_{2 k+1}=0 .
\end{aligned}
$$

\subsubsection{Cartesian coordinate}

The discharge electric field has been chosen in the $x$-direction and the $y z$-plane has been proposed as the symmetry plane of the system. So, the flat electrodes have been placed at $x=0$ and $x=L$ and each one's width is from $y=-Y_{0}$ to $y=Y_{0}$. By this arrangement, the equation (12) will be changed to the following form:

$$
\begin{aligned}
& \frac{\mathrm{d}^{2} n_{\mathrm{e}}}{\mathrm{d} y^{2}}+\beta n_{\mathrm{e}}-\eta^{2} n_{\mathrm{e}}^{2}=0 \\
& \Rightarrow \frac{\mathrm{d}^{2} g(z)}{\mathrm{d} y^{2}}-\left(\frac{\beta}{2}\right)\left(g^{2}(y)-1\right)=0 .
\end{aligned}
$$


Here, $g(y)=\left(\frac{2 \eta^{2} n_{\mathrm{e}}(y)}{\beta}-1\right)$ is a dimensionless function. By assuming the series formula like $g(y)=$ $\sum_{n=0}^{\infty} c_{n} y^{n}$ for the solution of this nonlinear ordinary differential equation and using the rules like what has been used in cylindrical coordinates the following formulas have been derived:

$$
\begin{aligned}
& \text { a) } c_{0}=\frac{2 \eta^{2}\left(n_{\mathrm{e}}\right)_{\max }}{\beta}-1=\frac{2+\sqrt{4+\beta^{2}}}{\beta}, c_{1}=0 \\
& \text { b) } c_{n+2}=\frac{\beta / 2}{(n+2)(n+1)} d_{n}, d_{n}=\sum_{j=0}^{n} c_{n-j}^{*} c_{j},
\end{aligned}
$$

where $n=1,2,3, \ldots$ The definition of $d_{n_{0}}$, likewise $b_{n_{0}}$ in the preceding section, is a second order nonlinear term, which has been obtained from inserting the series definition of the $g(y)$ in the equation (18). The expression $\left(n_{\mathrm{e}}\right)_{\max }$ is the maximum electron density of the discharge, which is taken into account on the $y$-axis. In this case, using the equation (18) and by intention to make the function $g(y)$ dimensionless, the following equation for the solution has been resulted:

$$
\begin{aligned}
g(y) & =G\left(\beta y^{2}\right)=c_{0}+\sum_{k=1}^{\infty} \frac{c_{2 k-2}}{2(k+1)(k+2)}\left(\frac{\beta y^{2}}{2}\right)^{k} \\
c_{1} & =c_{(2 k+1)}=0
\end{aligned}
$$

\subsection{Mathematical results and their physical interpretation}

In this section, the characteristic equation of the glow discharge has been derived by considering the condition of zero free charge density on the inner wall of the discharge vessel, $r=R_{0}$ from the central axis (cylindrical polar) or $y=Y_{0}$ from the symmetry axis (Cartesian coordinate):

$$
\begin{aligned}
& \text { a) } n_{\mathrm{e}}\left(R_{0}\right)=0 \Rightarrow f(r)=F\left(\beta R_{0}^{2}\right)=-1 \\
& \Rightarrow a_{0}+\sum_{k=1}^{\infty} \frac{b_{2 k-2}}{4(k+1)^{2}}\left(\frac{\beta R_{0}^{2}}{2}\right)^{k}=0,
\end{aligned}
$$

$$
\begin{aligned}
& \text { b) } n_{\mathrm{e}}\left(Y_{0}\right)=0 \Rightarrow g(y)=G\left(\beta Y_{0}^{2}\right)=-1 \\
& \Rightarrow c_{0}+\sum_{k=1}^{\infty} \frac{c_{2 k-2}}{2(k+1)(2 k+1)}\left(\frac{\beta Y_{0}^{2}}{2}\right)^{k}=0
\end{aligned}
$$

The physical condition $\eta^{2} n_{e} \leqslant \beta$ makes an interval for $a_{0}$ and $c_{0}$ where varies from near 1 (which corresponds to negligible or nearly zero recombination coefficient) to 1 (which corresponds to $\eta^{2} n_{\mathrm{e}} \approx \beta$ ). Then, the above equations (21a) and (21b) have been solved using the evolutionary genetic algorithm in MatLab ${ }^{\circledR}$, which results are shown in two figures in Figure 1 (a) and (b). The horizontal axis of figures has been calibrated in terms of the $\alpha$ parameter, which is defined
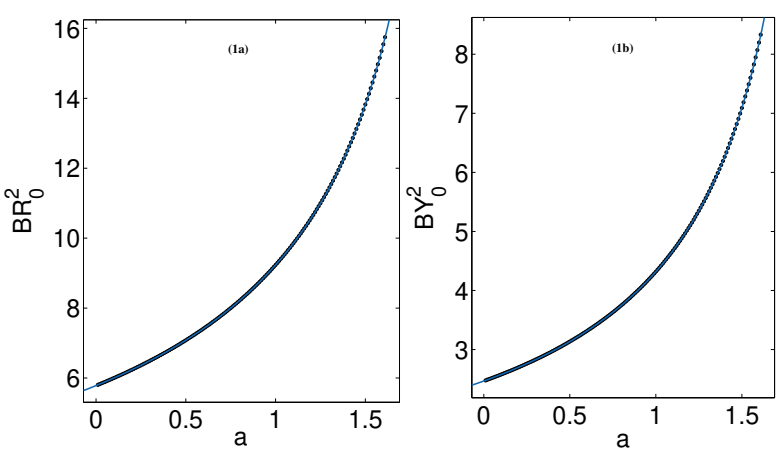

Figure 1. The diagrams of the solution of the equation (21a) in (a) Cylindrical Polar Coordinates and (21b) in (b) Rectangular Cartesian Coordinates vs. the $\alpha$ parameter where it is dependent to the electron density

as follow:

$$
\begin{aligned}
a_{0}+1=c_{0}+1 & =\frac{2 \eta^{2}\left(n_{\mathrm{e}}\right)_{\max }}{\beta} \\
& =2 \frac{\gamma}{R_{\mathrm{eff}}} \frac{\left(n_{\mathrm{e}}\right)_{\max }}{N}=\alpha,
\end{aligned}
$$

where $\frac{\left(n_{\mathrm{e}}\right)_{\max }}{N}$ is considered as the maximum degree of ionization which takes place on the axis of symmetry of the discharge. Figure (1) illustrate graphically the general dependence of the glow discharge electron temperature to the parameter $\alpha=2 \frac{\gamma}{R_{\text {eff }}} \frac{\left(n_{\mathrm{e}}\right)_{\max }}{N}$, which can be considered as a generalized characteristic figure of the glow discharge. Obviously, such reliance means the electron temperature has to be affected by electron density and current of the discharge which was observed experimentally before in pure media [29, 30].

Importantly, as the $\gamma$ approach zero, figure 1(a) shows that $\beta R_{0}^{2}$ tends to the common value of $\left(\beta R_{0}^{2}\right)_{\min }=(2.4)^{2}=5.76$, which corresponds to the glow discharge in cylindrical coordinate control by the ambipolar diffusion. In the case of figure $1(\mathrm{~b})$, the graph tends to the value $\left(\beta Y_{0}^{2}\right)_{\min }=(1.57)^{2}=2.4649$, which is related to the diffusion dominated glow discharge in the Cartesian coordinates. By the way, this has been proposed as a sign of the accuracy of the model, calculations and the resulted solutions. Given that, it can be shown that:

a) $\beta R_{0}^{2}=\left(N R_{\mathrm{eff}} / D_{\mathrm{a}}^{\mathrm{e}, \pm}\right) R_{0}^{2}$

$$
=\left(N R_{0}\right)^{2} \frac{R_{\mathrm{eff}}}{\frac{k_{\mathrm{B}}}{e}\left(T_{\mathrm{e}}+T_{\mathrm{i}}\right)}\left(\frac{1}{N \mu_{\mathrm{e}}}+\frac{1}{N \mu_{\mathrm{i}}}\right),
$$

b) $\beta Y_{0}^{2}=\left(N R_{\text {eff }} / D_{\text {a }}^{\mathrm{e}, \pm}\right) Y_{0}^{2}$

$$
=\left(N Y_{0}\right)^{2} \frac{R_{\mathrm{eff}}}{\frac{k_{\mathrm{B}}}{e}\left(T_{\mathrm{e}}+T_{\mathrm{i}}\right)}\left(\frac{1}{N \mu_{\mathrm{e}}}+\frac{1}{N \mu_{\mathrm{i}}}\right),
$$

where $k_{\mathrm{B}}$ and $e$ are Boltzmann constant and electron charge, respectively. So, from equation (23) and figure (1), it is obviously clear that the electron temperature in the generalized nonlinear model of the glow 
discharge will be dependent to the electron density, too.

Finally, by fitting the most consistent mathematical relation to discrete points, as shown in figure (2), the following equations have been obtained as a function of the general characteristic of the glow discharge:

$$
\begin{aligned}
\left.\begin{array}{l}
\beta R_{0}^{2}(x) \\
\beta Y_{0}^{2}(x)
\end{array}\right\}= & \frac{P_{1} x^{2}+P_{2} x+P_{3}}{x^{2}+Q_{1} x+Q_{2}}, \\
& \text { where } x=\frac{2 \eta^{2}}{\beta}\left(n_{\mathrm{e}}\right)_{\max } .
\end{aligned}
$$

The coefficients of the above mathematical equations in terms of the corresponding coordinate system are shown in Table (1). A valuable result that has come out of the mathematics was $\alpha_{\max } \leqslant 1$, and it means that for having a self-sustained DC glow discharge which is controlled by diffusion-recombination, the limit of $\frac{\gamma}{R_{\mathrm{eff}}} \frac{\left(n_{\mathrm{e}}\right)_{\max }}{N} \leqslant 0.5$ must be satisfied.

\begin{tabular}{ccc}
\hline \hline Coefficient & \multicolumn{2}{c}{ System of Coordinate } \\
& Cylindrical Polar & Cartesian Rectangular \\
\hline P1 & 0.1205 & 0.0059 \\
P2 & -16.57 & -5.884 \\
P3 & 39.35 & 13.39 \\
Q1 & -5.324 & -4.688 \\
Q2 & 6.803 & 5.429 \\
\hline \hline
\end{tabular}

Table 1. The parameters of the rational function in (24) in cylindrical and Cartesian coordinates.

Next, by replacing the axis of figures in Figure $1(\mathrm{a})$ and $1(\mathrm{~b})$, a graphical demonstration of the $\frac{2 \eta^{2} n_{\mathrm{e}}(x)_{\max }}{\beta}=\alpha(x)$ versus $\left\{\begin{array}{l}\beta R_{0}^{2} \\ \beta Y_{0}^{2},\end{array}\right.$ has been derived.

The results are shown in figures $2(\mathrm{a})$ and $2(\mathrm{~b})$.

The most consistent mathematical relations that are expressing the dependence of $n_{\mathrm{e}}(x)_{\max }$ on the geometrical and microscopic properties of the discharge in Cylindrical polar and Cartesian Rectangular coordinates, have been again fitted to the data and parameters of mathematical formula (25) have been found (see table 2).

$$
\begin{aligned}
n_{e}(x)_{\max } & =\frac{\beta}{2 \eta^{2}}\left(\frac{P_{1} x^{2}+P_{2} x+P_{3}}{x^{2}+Q_{1} x+Q_{2}}\right) \\
x & =\left\{\begin{array}{l}
\beta R_{0}^{2} \\
\beta Y_{0}^{2}
\end{array}\right.
\end{aligned}
$$

The coefficients of the equation (25) for the two systems of coordinate are given in Table (2).

Finally, using definition the spatially averaged electron density, $\bar{n}_{\mathrm{e}}=\frac{1}{\xi_{0}} \int_{0}^{\xi_{0}} n_{e}\left(\xi^{\prime}\right) d \xi^{\prime}$, where $\xi^{\prime}=\left(\begin{array}{l}r \\ y\end{array}\right)$ and $\xi_{0}=\left(\begin{array}{l}R_{0} \\ Y_{0}\end{array}\right)$, in equation (24) for cylindrical coordinates, and equation (25) for rectangular coordinates, the equation (26) is obtained for the dependence of
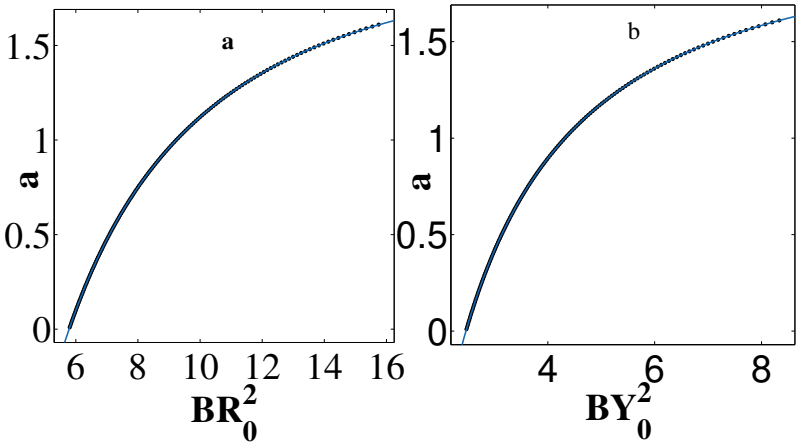

Figure 2. The diagrams of the $\alpha$ parameter where is dependent to the electron density, vs. $\beta R_{0}^{2}$ in (a) Cylindrical Polar Coordinates and vs. $\beta Y_{0}^{2}$ in (b) Rectangular Cartesian Coordinates which are results from equation (21)

\begin{tabular}{ccc}
\hline \hline Coefficient & \multicolumn{2}{c}{ System of Coordinate } \\
& Cylindrical Polar & Cartesian Rectangular \\
\hline P1 & 1.828 & 1.845 \\
P2 & 37.09 & 61.72 \\
P3 & 42.34 & 68.9 \\
Q1 & 16.37 & 28.29 \\
Q2 & 44.26 & 70.66 \\
\hline \hline
\end{tabular}

Table 2. The parameters of the rational function in (25) in cylindrical and Cartesian coordinates.

the (average) electrical conductivity of the plasma glow discharge [31-38] to the geometry, and electron and ionic temperatures.

$$
\begin{aligned}
& \bar{\sigma}=e \frac{\bar{n}_{\mathrm{e}}}{N}\left(N \mu_{\mathrm{e}}\right)=e\left(N \mu_{\mathrm{e}}\right) \\
& \times\left(\begin{array}{c}
\frac{\beta}{2 \eta^{2}}\left(\frac{n_{e}}{N}\right)_{\max }+\frac{R_{0}}{S V} \sum_{k=1}^{\infty} \frac{b_{2 k-2}}{4(k+1)^{2}(2 k+1)}(x / 2)^{k} \\
\frac{\beta}{2 \eta^{2}}\left(\frac{n_{e}}{N}\right)_{\max }+\frac{Y_{0}}{S V} \sum_{k=1}^{\infty} \frac{b_{2 k-2}}{2(k+1)(k+2)^{2}}(x / 2)^{k}
\end{array}\right), \\
& S V=\left(\begin{array}{c}
N R_{0}^{2} \\
N Y_{0}^{2}
\end{array}\right), \\
& x=\left(\begin{array}{c}
\beta R_{0}^{2} \\
\beta Y_{0}^{2}
\end{array}\right),
\end{aligned}
$$

where $\frac{\bar{n}_{e}}{N},\left(\frac{n_{e}}{N}\right)_{\max }$ are the average and maximum degree of ionization, and $N \mu_{\mathrm{e}}$ is electron reduced mobility (in gaseous medium), respectively. Also, $S V=\left(\begin{array}{c}N R_{0}^{2} \\ N Y_{0}^{2}\end{array}\right)$, is the similarity variable of the discharge in Cylindrical or Cartesian coordinate, The reason for using the above parameters is that the degree of ionization (average or maximum) irrespective of the type and amount of overall densities has values between 0 and up to 1 while $\left(\frac{n_{e}}{N}\right)_{\max } \geqslant \frac{\bar{n}_{e}}{N}$. The reduced mobility of electron $N \mu_{\mathrm{e}}$, is independent of the density of the environment and depend only on the 
composition of the medium [2]. After all, the above equations depict the average of the electrical conductivity of the positive column of the glow discharge can be specified based on the small-scale properties (electron-ion temperature and ionization rates and maximum degree of ionization of the medium) and the large scale (geometric dimensions of the chamber and particle density i.e., similarity variable). So, by measuring the electric field intensity and current density of the positive column of the glow discharge and having a specified similarity variable the electron temperature will be simply estimated.

\section{Conclusion}

By including in the complementary process, metastable excited states ionization, in the diffusionrecombination dominated glow discharge in a single component gaseous, or metal vapor, media an improved system of the nonlinear time-independent continuity equation of the electron and ions (positive and negative) have been obtained. It has been shown that by proposing some physical estimation, a nonlinear partial differential equation of the charged particle density was obtained. By simplification of the obtained equation in the Cylindrical (polar) and the Cartesian (rectangular) system of coordinates, the corresponding nonlinear ordinary equations have been derived. Then by using a series based solution, the innovative non-linear recursion equations have been obtained for establishing the coefficients of the series.

By imposing two physical conditions, namely the maximum charge density on the symmetry axis, or plane, and removing of free charge at the discharge tank boundaries, the glow discharge characteristic equation is obtained, expressing the dependence of electron characteristic energy on the similarity variable and free charge density variable. Adaptation of the results to the familiar situation, when the model is close to the simple ambipolar diffusion dominated regime in both coordinate systems, can be considered as a test for the accuracy of the introduced physicalmathematical model. Despite the limitation for maximum acceptable recombination in the positive column of a self-sustained glow discharge, the relations can be considered as a new physical-mathematical equation in the subject of electrical gas discharge.

In the last part, the average plasma conductivity of the positive column of the glow discharge has been defined according to the electron temperature, and similarity variable. It has been insisted that this equation provide a simple method for estimating the electron temperature by measuring the electric field intensity and current density of the positive column.

Unfortunately, no other theoretical research results or unique experimental reference were found for comparison which would shows the nonlinear nature of the relation of the $k T_{\mathrm{e}}$ and similarity variable of the glow discharge in the $\mathrm{CO}_{2}$ laser.
Extending the introduced nonlinear model to the glow discharge in the mixture of atomic and molecular gases is left for the next step.

\section{Acknowledgements}

The advises of Prof. G. Colonna about having the superelastic collision of the electrons and metastable states in the model and the kindful help of Dr. Petr Kloc in precise editing of the LaTex form of the article is greatly appreciated.

\section{References}

[1] B. M. Smirnov. Theory of Gas Discharge Plasma. Springer International Publishing, 2014. ISBN 978-3-319-11064-6.

[2] B. E. Cherrington. Gaseous Electronics and Gas Lasers. Pergamon Press, 2014. ISBN 978-1-483-23400-7.

[3] Y. P. Raizer. Gas Discharge Physics. 1st Edition reprint. Springer-Verlag Berlin Heidelberg, 2011. ISBN 978-3-642-64760-4.

[4] M. P. Jahan, editor. Electrical Discharge Machining (EDM): Types, Technologies and Applications. Nova Science Publishers, Incorporated, 2015. ISBN 978-1-634-83591-6.

[5] M. C. Jones, D. J. Ampleford, M. E. Cuneo, et al. $\mathrm{X}$-ray power and yield measurements at the refurbished $\mathrm{Z}$ machine. Review of Scientific Instruments, 85(8):083501, 2014. doi:10.1063/1.4891316.

[6] M. Thompson. Handbook of Inductively Coupled Plasma Spectrometry. 2nd Edition. Springer US, 2012. ISBN 978-1-4612-8037-8.

[7] G. Colonna and A. D'Angola, editors. Plasma Modeling, Methods and Applications. IOP Publishing Ltd, 2016. ISBN 978-0-7503-1200-4.

[8] M. Aram, N. Morshedian, S. Behrouzinia, and M. Namnabat. An innovative simple method for study of the characteristics of the trigatron plasma switch. Contributions to Plasma Physics, 56(10):982-986, 2016. doi:10.1002/ctpp. 201600023.

[9] A. Bogaerts, E. Neyts, R. Gijbels, and J. van der Mullen. Gas discharge plasmas and their applications. Spectrochimica Acta Part B: Atomic Spectroscopy, 57(4):609-658, 2002.

doi: 10.1016/S0584-8547(01)00406-2.

[10] A. Bogaerts and R. Gijbels. Fundamental aspects and applications of glow discharge spectrometric techniques. Spectrochimica Acta Part B: Atomic Spectroscopy, 53(1):1-42, 1998. doi:10.1016/S0584-8547(97)00122-5.

[11] M. R. Kenneth, editor. Glow Discharge Spectroscopies. Springer US, 2013. ISBN 978-1-489-92396-7.

[12] W. J. Witteman, editor. The $\mathrm{CO}_{2}$ Laser. 1st Edition reprint. Springer-Verlag Berlin Heidelberg, 2013. ISBN 978-3-662-13617-1.

[13] D. Basting and G. Marowsky, editors. Excimer Laser Technology. Springer-Verlag Berlin Heidelberg, 2005. ISBN 978-3-642-05749-6. 
[14] T. J. Killian. The uniform positive column of an electric discharge in mercury vapor. Physical Review, 35(10):1238-1252, 1930.

doi:10.1103/PhysRev.35.1238.

[15] V. Granovsky. On the applicability of similarity laws to the positive column in mercury discharges. Proceedings of the USSR Academy of Science, 28:37, 1940.

[16] B. A. Anicin. A theory of the low-pressure positive column in a transverse magnetic field. The Physics of Fluids, 10(11):2377-2383, 1967. doi:10.1063/1.1762046.

[17] K. Chandrakar. Electron temperature in an a.c. glow discharge in air. British Journal of Applied Physics, 16(4):449-451, 1965. doi:10.1088/0508-3443/16/4/305.

[18] U. Kortshagen and L. D. Tsendin, editors. Electron Kinetics and Applications of Glow Discharges. Springer US, 2006. ISBN 978-0-306-47076-9.

[19] S. Ono and S. Teii. Negative ion formations and their effects on the electron temperature in $\mathrm{CO}_{2}-\mathrm{N}_{2}$-he mixture gas discharges. Journal of Physics D: Applied Physics, 17(10):1999-2008, 1984. doi : 10.1088/0022-3727/17/10/011.

[20] C. Leys, C. van Egmond, and E. Desoppere. Ionization equilibrium in flowing $\mathrm{CO}_{2}$ laser mixtures. Journal of Physics D: Applied Physics, 30(4):573-581, 1997. doi:10.1088/0022-3727/30/4/011.

[21] H. T. Davis. Introduction to Nonlinear Differential and Integral Equations. 1st Edition. U.S. Atomic Energy Commission, 1960.

[22] A. Plastino and M. C. Rocca. From the hypergeometric differential equation to a non-linear schrödinger one. Physics Letters A, 379(42):2690-2693, 2015. doi:10.1016/j.physleta.2015.08.015.

[23] J. Guckenheimer and P. J. Holmes. Nonlinear Oscillations, Dynamical Systems, and Bifurcations of Vector Fields. Corrected 7th Edition. Springer-Verlag New York, 2013. ISBN 978-1-4612-1140-2.

[24] A. S. Al-Johani and M. A.-A. El-Beltagy. Numerical solution of stochastic nonlinear differential equations using wiener-hermite expansion. In Proceedings of the International Conference on Numerical Analysis and Applied Mathematics 2013 (ICNAAM 2013), volume 1558 of AIP Conference Proceedings, Rhodes, Greece, 2013. AIP Publishing. ISBN 978-0-7354-1184-5.

[25] C. V. Pao. Nonlinear Parabolic and Elliptic Equations. Springer US, 2012. ISBN 978-1-461-53034-3.

[26] H. K. Avetissian. Relativistic Nonlinear Electrodynamics: Interaction of Charged Particles with Strong and Super Strong Laser Fields, volume 120 of Springer Series in Optical Sciences. Springer-Verlag New York, 2007. ISBN 978-1-4419-2135-2.

[27] B.-Y. Guo and C.-L. Xu. Hermite pseudospectral method for nonlinear partial differential equations. ESAIM: Mathematical Modelling and Numerical Analysis, 34(4):859-872, 2000. doi:10.1051/m2an:2000100.
[28] J. R. de Oliveira and M. A. de Moura. Analytical solution for the modified nonlinear Schrödinger equation describing optical shock formation. Physical Review E, 57(4):4751-4756, 1998. doi:10.1103/PhysRevE.57.4751.

[29] W. Elenbaas. Der gradient der quecksilber-hochdruckentladung als funktion von druck, durchmesser und stromstärke. Physica, 2(1-12):787-792, 1935. doi:10.1016/S0031-8914(35)90158-6.

[30] A. Lompe, R. Seeliger, and E. Wolter. Untersuchungen an Hohlkathoden. Annalen der Physik, 428(1):9-37, 1939. doi:10.1002/andp.19394280103.

[31] R. Radtke and K. Gunther. Electrical conductivity of highly ionized dense hydrogen plasma. I. Electrical measurements and diagnostics. Journal of Physics D: Applied Physics, 9(7):1131-1138, 1976. doi : 10.1088/0022-3727/9/7/007.

[32] G. E. Norman and A. A. Valuev. Electrical conductivity of nonideal plasma. Plasma Physics, 21(6):531-544, 1979. doi:10.1088/0032-1028/21/6/002.

[33] Y. K. Kurilenkov and A. A. Valuev. The electrical conductivity of plasma in wide range of charge densities. Beiträge aus der Plasmaphysik, 24(3):161-171, 1984. doi:10.1002/ctpp. 19840240304.

[34] M. R. Zaghloul, M. S. Al Na'imi, and M. A. Bourham. Measurement of electrical conductivity of weakly nonideal multicomponent plasma mixtures generated from dielectric materials. IEEE Transactions on Plasma Science, 37(8):1626-1631, 2009. doi:10.1109/TPS. 2009.2024423.

[35] R. J. Zollweg and R. W. Liebermann. Electrical conductivity of nonideal plasmas. Journal of Applied Physics, 62(9):3621-3627, 1987. doi:10.1063/1.339265.

[36] T. Ramazanov, K. Galiyev, K. N. Dzhumagulova, et al. Scattering processes and electrical conductivity of partially ionized hydrogen plasma. Contributions to Plasma Physics, 43(1):39-46, 2003. doi:10.1002/ctpp. 200310005

[37] M. Baus, J.-P. Hansen, and L. Sjögren. Electrical conductivity of a strongly coupled hydrogen plasma. Physics Letters A, 82(4):180-182, 1981. doi : 10.1016/0375-9601(81)90115-8.

[38] B. Chervy and A. Gleizes. Electrical conductivity in $\mathrm{SF}_{6}$ thermal plasma at low temperature (1000-5000 K). Journal of Physics D: Applied Physics, 31(19):25572565, 1998. doi:10.1088/0022-3727/31/19/029. 